\title{
Competition for light in forest population dynamics: from computer simulator to mathematical model
}

\author{
Pierre Magal ${ }^{1}$ \\ Zhengyang Zhang ${ }^{1}$
}

1. Univ. Bordeaux, IMB, UMR 5251, F-33076 Bordeaux, France; CNRS, IMB, UMR 5251, F-33400 Talence, France.

Keywords: Computer forest simulator, SORTIE model, size structured model, spatial structured model, state dependent delay differential equations.

Manuscript type: Article. 


\section{Abstract}

In this article we build a mathematical model for forest growth and we compare this model 3 with a computer forest simulator named SORTIE. The main ingredient taken into account in both models is the competition for light between trees. The parameters of the mathematical model are estimated by using SORTIE model, when the parameter values of SORTIE model correspond

6 to the ones previously evaluated for the Great Mountain Forest in USA. We construct a size structured population dynamics model with one and two species and with spatial structure. 


\section{Introduction} ple to propose computer simulators as well as mathematical models to describe the dynamical properties of forests. Many computer simulators (also sometimes called Individual Based Models (IBMs)) have been proposed and we refer to JABOWA ([3, 2]), FORET [25], SORTIE [19], FORMIND [9] and others. These models consist of stochastic processes describing individual behaviors, such as birth, death, movement, reproduction and so on. Moreover, these models also permit to describe the behavior of the entire plant community. The main advantage is that it provides simulated data which can be used to analyze such a complex system. Of course this is a rough description of the real plant community. However, they do supply powerful experimental tools and describe the forest dynamics reasonably well [13]. We refer to [14, 21] for a general review about forest IBMs.

SORTIE is a forest simulator based on the forest data observed in and around Great Mountain Forest (GMF), a privately owned 2500ha forest located in northwestern Connecticut $\left(41^{\circ} 57^{\prime} \mathrm{N}\right.$, $\left.73^{\circ} 15^{\prime} \mathrm{W}\right)$, USA in the year 1990-1992. In SORTIE, four submodels (resource, growth, mortality, and recruitment) are included to determine the behaviour of each individual. As is explained in [19], SORTIE includes only the light limitation as the competition for resources, since little evidence of the effects of water or nitrogen on the growth has been found after extra experiments are performed. Tree growth is described by change of tree size, which is denoted here as the diameter at a certain height. Two concepts "diam 10 (Diameter at $10 \mathrm{~cm}$ Height)" and "DBH (Diameter at Breast Height)" are often used to describe the tree growth and represent the tree size in the analysis of forest dynamics $([19,22,18])$. Thereinto, the diam 10 can be used almost throughout the whole life of an individual, from seedling to adult, while DBH can only be used for adults in most cases, as it is measured at a higher height. The definition of the breast height (of an adult human being) is different in different regions, for example, $1.4 \mathrm{~m}$ in the US and $1.3 \mathrm{~m}$ in Europe and Canada. But it makes little difference to the measuring result in many cases. We

In the natural ecosystem, forests play an important role. This has motivated a lot of peo- 
refer to $[29,19,20,22,8,18]$ for more details of SORTIE.

In this article we will extend the model proposed by Hal Smith in [26, 27] to describe the numerical simulations to see the spread of trees in space. 


\section{Single species model}

\section{Mathematical modelling}

In this section we consider the following model describing the growth of trees of single species

$$
\left\{\begin{array}{l}
\partial_{t} u(t, s)+\underbrace{f(A(t)) \partial_{s} u(t, s)}_{\text {growth of adults }}=-\underbrace{\mu(s)}_{\text {mortality }} u(t, s), \text { for } t>0, s>s_{-}, \\
f(A(t)) u\left(t, s_{-}\right)=\underbrace{\beta b(A(t))}_{\text {flux of newborns }}, \text { for } t>0, \\
u(0, .)=u_{0}(.) \in L^{1}(0,+\infty) .
\end{array}\right.
$$

Here $u(t, s)$ denotes the population density of trees with size $s$ at time $t$, so $\int_{s_{1}}^{s_{2}} u(t, s) d s$ is the number of trees with size $s \in\left[s_{1}, s_{2}\right]$ at time $t$, and $A(t)$ is the number of adult population at time $t$. The size $s$ is described by a function of $\operatorname{diam}_{10}$, which we will see in the appendix.

The function $\mu(s)>0$ is the natural mortality. The minimal size of a juvenile is denoted as $s_{-}$. The parameter $\beta$ is the birth rate in absence of birth limitation, and the term $\beta b(A(t))$ describes the flux of newborns into the population, where $b(x)=x e^{-\xi x}$ is the Ricker's type birth limitation $([23,24])$. The growth function $f(x)$ takes the form

$$
f(x)=\frac{\alpha}{1+\delta x}, \alpha, \delta>0
$$

which is decreasing, thus taking care of the fact that the more large trees there are, the slower the growth rate of small trees is. So this shows the type of competition for light between adults and juveniles. The function $u_{0}($.$) represents the initial distribution of the species. Normally we want$ the number of the total population to be finite at each time, hence we have

$$
\int_{0}^{+\infty} u(t, s) d s<+\infty, \forall t \geqslant 0
$$

So the natural state space for this model is $L^{1}$.

We will derive the following equations for adults and juveniles under some assumptions: 


$$
\left\{\begin{array}{l}
\frac{d A(t)}{d t}=f(A(t)) j\left(t, s^{*}\right)-\mu_{A} A(t), \text { for } t>0, \\
\partial_{t} j(t, s)+f(A(t)) \partial_{s} j(t, s)=-\mu_{J} j(t, s), \text { for } s \in\left[s_{-}, s^{*}\right), t>0 \\
f(A(t)) j\left(t, s_{-}\right)=\beta b(A(t)), \text { for } t>0, \\
A(0)=A_{0} \geqslant 0, \\
j(0, s)=j_{0}(s) \geqslant 0, \text { for } s \in\left[s_{-}, s^{*}\right)
\end{array}\right.
$$

where $j(t, s)$ represents the population density of juveniles with size $s \in\left[s_{-}, s^{*}\right)$ at time $t$, and the positive constant $s^{*}$ satisfying $s^{*}>s_{-}$denotes the maximal size of a juvenile (or the minimal size of an adult). Hence the total number of juveniles at time $t$ is

$$
J(t)=\int_{s_{-}}^{s^{*}} j(t, s) d s=\int_{s_{-}}^{s^{*}} u(t, s) d s .
$$

And we can assume as follows the adult population number

$$
A(t)=\int_{s^{*}}^{+\infty} u(t, s) d s
$$

By integrating along the characteristic line of the second equation (of juvenile) in (0.3), the first equation (of adult) in (0.3) can be rewritten as the following state dependent Functional Differential Equation (FDE)

$$
\left\{\begin{array}{l}
\frac{d A(t)}{d t}=e^{-\mu_{J} \tau(t)} \frac{f(A(t))}{f(A(t-\tau(t)))} \beta b(A(t-\tau(t)))-\mu_{A} A(t), \\
\int_{t-\tau(t)}^{t} f(A(\sigma)) d \sigma=s^{*}-s_{-}
\end{array}\right.
$$

when $t>t^{*}$, where $t^{*}$ is defined as

$$
\int_{0}^{t^{*}} f(A(\sigma)) d \sigma=s^{*}-s_{-}
$$

Differentiation of the second equation with respect to $t$ gives the following system

$$
\left\{\begin{array}{l}
A^{\prime}(t)=e^{-\mu_{J} \tau(t)} \frac{f(A(t))}{f(A(t-\tau(t)))} \beta b(A(t-\tau(t)))-\mu_{A} A(t), \\
\tau^{\prime}(t)=1-\frac{f(A(t))}{f(A(t-\tau(t)))} .
\end{array}\right.
$$

The initial conditions are

$$
A(t)=A_{0}(t) \geqslant 0, \forall t \in(-\infty, 0] ; \tau(0)=\tau_{0} \geqslant 0,
$$


where $A_{0}(t)$ is continuous and exponentially bounded, namely for some $\vartheta>0$

$$
\sup _{t \leqslant 0} e^{\vartheta t} A_{0}(t)<+\infty
$$

From the second equation of (0.5), as $f$ is decreasing, the delay $\tau(t)$ can become large enough, namely we may have infinite delay. For all the derivation here, see Appendix A.

\section{Numerical simulations of two special cases}

We conduct numerical simulations for two special cases of the system (0.5).

Special case $1(f(x)$ is constant): Assume that $f(x)$ is a constant function (so the delay $\tau(t)$ is also constant by the second equation of (0.5)) and $b(x)=x e^{-x}$. Then since the PDE model (0.1) can be transformed (by making a simple change of variable in time) into an age structured model, it is known (see Magal and Ruan [16]) that the system has a Hopf bifurcation around the positive equilibrium when $\beta$ increases (see in Figure 1).
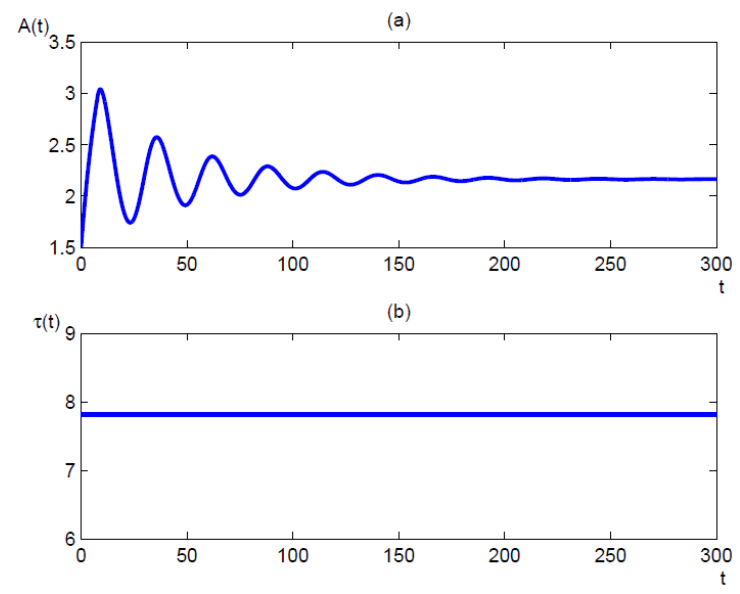
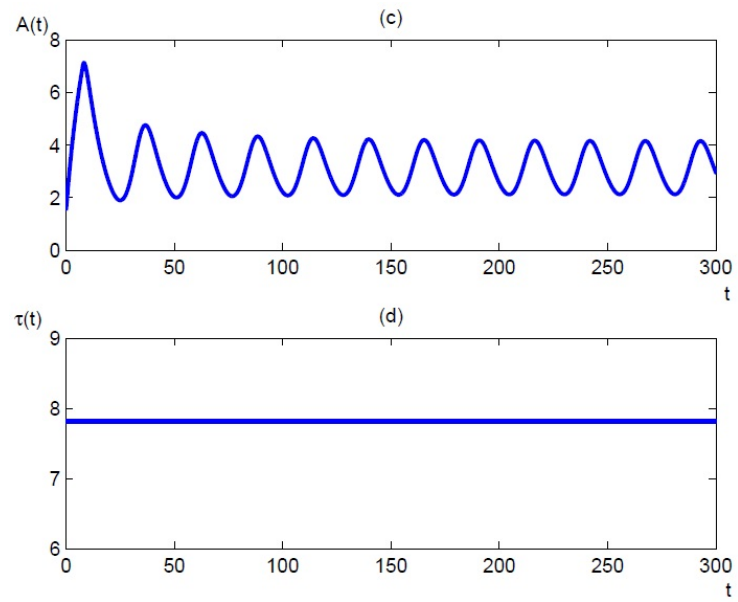

Figure 1: We plot the adult population number $A(t)$ in figure (a) and (c), and the corresponding delay $\tau(t)$ in figure (b) and $(d)$. We fix the parameter values $\mu_{J}=0.2, \mu_{A}=0.1, \alpha=0.5, \delta=0, \xi=1$, $s^{*}=\ln 50, s_{-}=0$, and the initial distribution $\phi(t)=1.5, \forall t \in[-100,0]$. In (a) and (b) we set $\beta=9$. The solution oscillates and then converges to the positive equilibrium. In (c) and (d) we set $\beta=25$. Changing $\beta$ from 9 to 25, we observe a Hopf bifurcation.

Special case $2(b(x)=x)$ : Assume $b(x)=x$, namely the Ricker's type birth function doesn't 
appear in system (0.5). It is known that when $\tau$ is constant in the first equation (which becomes linear) of system (0.5), this system is either exponentially increasing or exponentially decreasing when the time goes to infinity. However, it has been proved by Smith [26] that Hopf bifurcation can occur when we take state-dependent delay. This is illustrated in Figure 2.
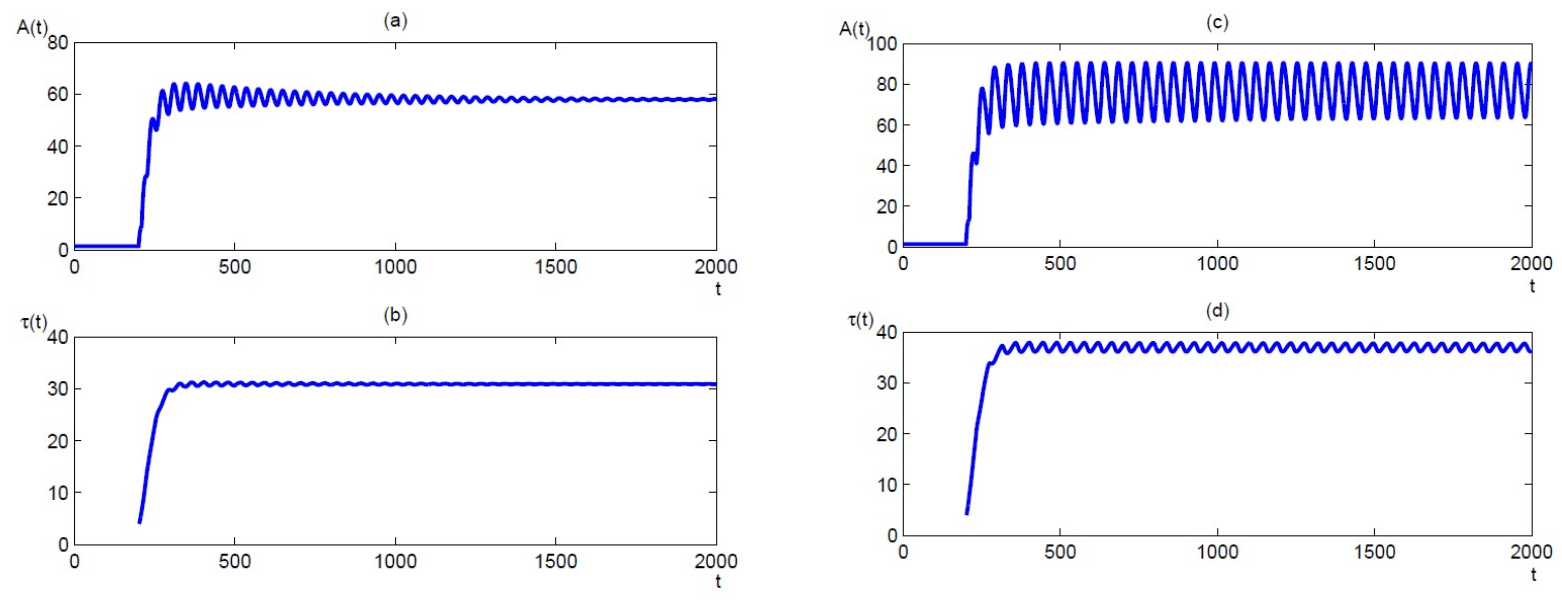

Figure 2: We plot the adult population number $A(t)$ in figure (a) and (c), and the corresponding delay $\tau(t)$ in figure (b) and (d). We fix the parameter values $\mu_{J}=\mu_{A}=0.1, \delta=0.1, \xi=0$ (remember this means that $b(x)=x$, and the initial delay $\tau_{0}=4$. The initial distribution is $\phi(t)=1.5, \forall t \in[0,200]$. In (a) and (b) we set $\beta=2.2$, then we have the damped oscillating solution which converges to the positive equilibrium; In (c) and (d) we set $\beta=4$. Changing $\beta$ from 2.2 to 4, we observe a Hopf bifurcation.

\section{Comparison with SORTIE}

We run the simulator SORTIE with the parameter values given in $[29,19,22,8]$ and get the simulation for the density of adult trees (adults are defined here as trees having a $\mathrm{DBH} \geqslant 10 \mathrm{~cm}$ ). And as we can see from this simulation, American beech(FAGR) and eastern hemlock(TSCA) become the dominant species after a period time. So in this article we will focus on these two species in two cases: one single species and two-species.

The basic idea of the numerical simulation of (0.5) and comparison is as follows. Before starting, we need to get the forest data from SORTIE. Since every run of SORTIE is initiated with a random seed, we conduct 50 runs and take the average values as our actual data. Moreover, the 
data that SORTIE gives are actually the density of the adult population per hectare. As the area of the sample square is $90000 \mathrm{~m}^{2}$ (a square of $\left.300 \mathrm{~m} \times 300 \mathrm{~m}\right)=9$ hectares, we multiply the data by 9 to obtain the total adult population number. We plot them and the average in MATLAB.

Now we will compare our model (0.5) with the mean value over these 50 runs of SORTIE, and find the best fit. First we need to decide the initial time (for example, $t=100$ as the initial time), and we will use the data from SORTIE over the time interval $[0,100]$ as the initial condition. Next we discretize the parameters $\mu_{J}, \mu_{A}, \beta, \xi, \delta, \tau_{0}$, and for each set of parameters, we calculate the solution of (0.5) by using the common approximation of the derivative (the numerical scheme will be conducted via the equivalent system (0.6)), and we compare the numerical solutions with the data from SORTIE by using the least square method, to find the set of parameter values with which the numerical result of the model (0.5) and the data have the least difference. Then we use the following formula (see Appendix A)

$$
\int_{-\tau_{0}}^{0} \frac{\alpha}{1+\delta A(\sigma)} d \sigma=s^{*}-s_{-},
$$

to compute $\alpha$, where we use the Simpson's rule to calculate the integral. Now we can keep this set of parameter values, and we have the best fit to SORTIE.

For the first dominant species American beech, we choose the SORTIE data in the time interval $[0,200]$ as the initial distribution. We have the best fit in Table 1 and Figure 3.
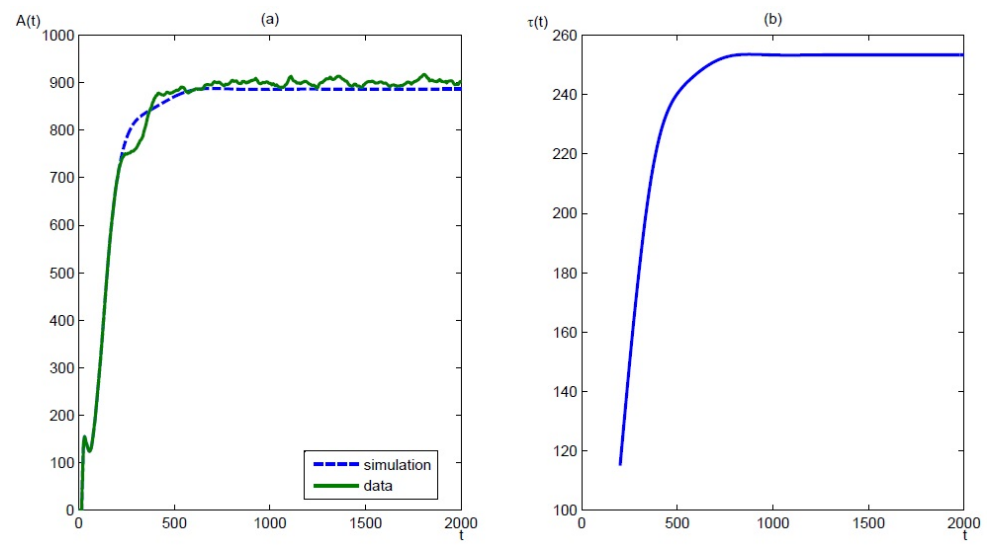

Figure 3: In this figure we show the comparison between SORTIE data and numerical simulation for American beech. The adult population number $A(t)$ is plotted in (a) and the delay $\tau(t)$ is shown in $(b)$. 
Similarly, for the second dominant species eastern hemlock we choose the SORTIE data in the time interval $[0,180]$ as the initial distribution. We get the best fit in Table 2 and Figure 4.
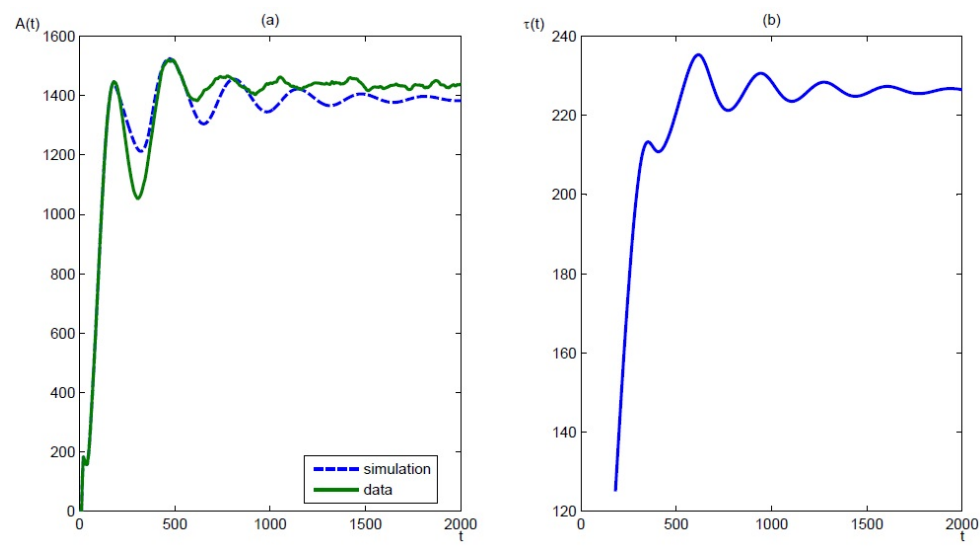

Figure 4: In this figure we show the comparison between SORTIE data and numerical simulation for eastern hemlock. The adult population number $A(t)$ is plotted in (a) and the delay $\tau(t)$ is shown in $(b)$. Notice that this species will have an oscillation before it converges to the stable positive equilibrium.

Notice that for both species in the single species case, we have $\xi_{1}=\xi_{2}=0$ as the best fit, which means that there is no Ricker's type birth limitation here.

\section{Two-species model}

\section{Mathematical modelling}

System (0.1) can be extended to the case of two species. Taking the previous best fit $\xi_{1}=\xi_{2}=$ 0 into account, we obtain the following system 


$$
\left\{\begin{array}{l}
\partial_{t} u_{1}(t, s)+f_{1}\left(Z_{1}(t)\right) \partial_{s} u_{1}(t, s)=-\mu_{1}(s) u_{1}(t, s), \text { for } t>0, s>s_{-}, \\
\partial_{t} u_{2}(t, s)+f_{2}\left(Z_{2}(t)\right) \partial_{s} u_{2}(t, s)=-\mu_{2}(s) u_{2}(t, s), \text { for } t>0, s>s_{-}, \\
f_{1}\left(Z_{1}(t)\right) u_{1}\left(t, s_{-}\right)=\beta_{1} A_{1}(t), \text { for } t>0 \\
f_{2}\left(Z_{2}(t)\right) u_{2}\left(t, s_{-}\right)=\beta_{2} A_{2}(t), \text { for } t>0 \\
u_{1}(0, \cdot)=u_{10}(\cdot) \in L^{1}(0,+\infty) \\
u_{2}(0, \cdot)=u_{20}(\cdot) \in L^{1}(0,+\infty)
\end{array}\right.
$$

where

$$
Z_{i}(t)=\zeta_{i 1} A_{1}(t)+\zeta_{i 2} A_{2}(t), f_{i}(x)=\frac{\alpha_{i}}{1+\delta_{i} x}, \mu_{i}(s)=\left\{\begin{array}{l}
\mu_{A_{i}}>0, \text { if } s \geqslant s^{*} \\
\mu_{J_{i}}>0, \quad \text { if } s \in\left[s_{-}, s^{*}\right)
\end{array}\right.
$$

and $\zeta_{i j} \geqslant 0$ are nonnegative constants, $\alpha_{i}, \delta_{i}>0, i, j=1,2$. Notice that we use the same minimal juvenile size $s_{-}$and minimal adult size $s^{*}$ for both species([19]). After a similar derivation, we have the following state-dependent delay differential equations

$$
\left\{\begin{array}{l}
A_{i}^{\prime}(t)=e^{-\mu_{J_{i}} \tau_{i}(t)} \frac{f_{i}\left(Z_{i}(t)\right)}{f_{i}\left(Z_{i}\left(t-\tau_{i}(t)\right)\right)} \beta_{i} A_{i}\left(t-\tau_{i}(t)\right)-\mu_{A_{i}} A_{i}(t), \\
\int_{t-\tau_{i}(t)}^{t} f_{i}\left(Z_{i}(t)\right) d \sigma=s^{*}-s_{-},
\end{array}\right.
$$

$i=1,2$. We give the following expression for the sake of numerical simulation:

$$
\left\{\begin{array}{l}
A_{i}^{\prime}(t)=e^{-\mu_{J_{i}} \tau_{i}(t)} \frac{f_{i}\left(Z_{i}(t)\right)}{f_{i}\left(Z_{i}\left(t-\tau_{i}(t)\right)\right)} \beta_{i} A_{i}\left(t-\tau_{i}(t)\right)-\mu_{A_{i}} A_{i}(t), \\
\tau_{i}^{\prime}(t)=1-\frac{f_{i}\left(Z_{i}(t)\right)}{f_{i}\left(Z_{i}\left(t-\tau_{i}(t)\right)\right)}
\end{array}\right.
$$

\section{Comparison with SORTIE}

We use the same method of comparison as before and we use the parameters in Table 1 and Table 2 to simulate the two-species model. We discretize the new parameters $\zeta_{i j}$ appeared in the competition term, and also by the least square method, we get the best fit for them: $\zeta_{11}=1$, $\zeta_{12}=0.6, \zeta_{21}=1.6, \zeta_{22}=1$, and the delay $\tau_{01}$ is increased to $201, \tau_{02}$ increased to 208 . We list these values in Table 3. The comparison figure is in Figure 5. 

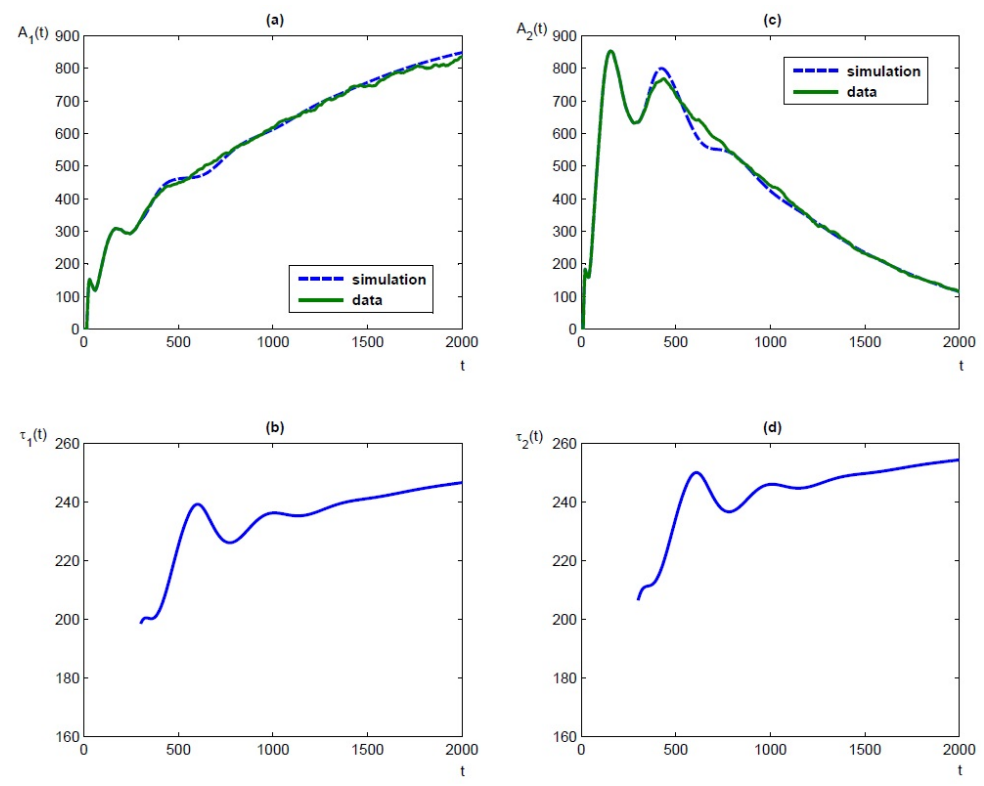

Figure 5: In this figure we plot the comparison between SORTIE data and numerical simulation for twospecies model (0.9). The figures (a) and (c) show the adult population number for species 1 American beech and species 2 eastern hemlock respectively. The figures $(b)$ and $(d)$ show the corresponding time delay.

By analyzing the existence positive (coexisting) equilibrium (see Appendix B), we can also obtain the coexistence of both American beech and eastern hemlock. 

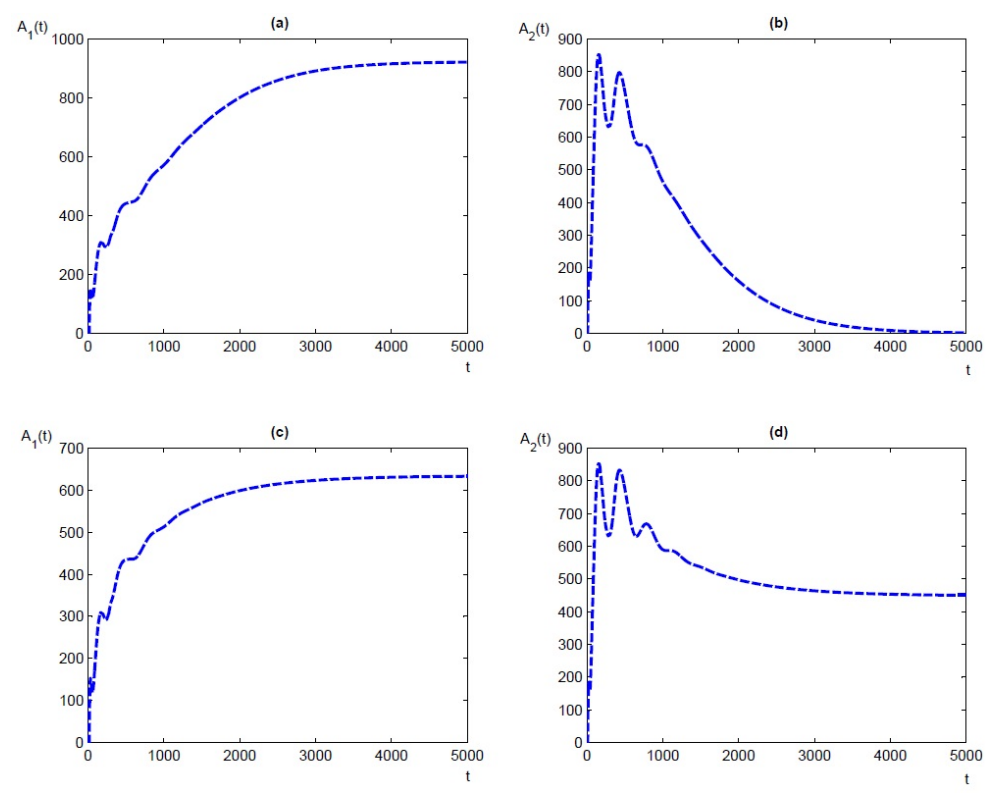

Figure 6: In this figure we demonstrate that we can pass from competitive exclusion $(a)(b)($ which corresponds to Figure 5) to coexistence $(c)(d)$ by changing one parameter $\zeta_{21}$. The other parameters are the same as in Figure 5. When $\zeta_{21}=1.6$, American beech (a) reaches to a positive steady state while eastern hemlock (b) disappears gradually. After we decrease the value of $\zeta_{21}$ to 1 , both American beech (c) and eastern hemlock (d) go to a positive steady state, which means coexistence.

\section{Two-species spatial model}

Now we take the spatial position of the individuals into account to see the spread of the adult population. Inspired by Ducrot [7], we can describe the spreading of the seeds around the adult trees by using the following two species model 


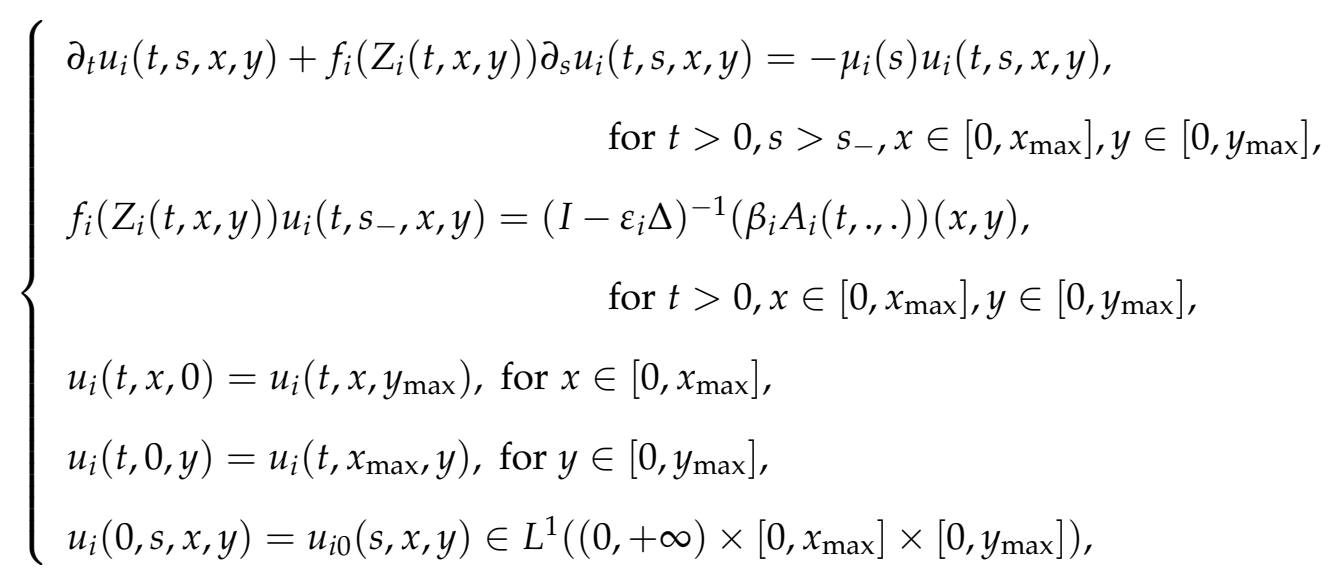

where

$$
Z_{i}(t, x, y)=\zeta_{i 1} A_{1}(t, x, y)+\zeta_{i 2} A_{2}(t, x, y), f_{i}(x)=\frac{\alpha_{i}}{1+\delta_{i} x}
$$

$\zeta_{i j} \geqslant 0, \alpha_{i}, \delta_{i}>0, i=1,2$, and $\Delta$ is the Laplacian operator with periodic boundary condition. Similarly, we assume the adult population number

$$
A_{i}(t, x, y)=\int_{s^{*}}^{+\infty} u_{i}(t, s, x, y) d s, i=1,2
$$

and by following a similar procedure as before, we get the state-dependent delay differential equation for the adult

$$
\left\{\begin{array}{c}
\frac{\partial A_{i}(t, x, y)}{\partial t}=e^{-\mu_{i} \tau_{i}(t, x, y)} \frac{f_{i}\left(Z_{i}(t, x, y)\right)}{f_{i}\left(Z_{i}\left(t-\tau_{i}(t, x, y), x, y\right)\right)}\left(I-\varepsilon_{i} \Delta\right)^{-1}\left(\beta_{i} A_{i}(t-\right. \\
\left.\left.\tau_{i}(t, x, y), ., .\right)\right)(x, y)-\mu_{A_{i}} A_{i}(t, x, y), \text { for } t>t^{*} \\
\int_{t-\tau_{i}(t, x, y)}^{t} f_{i}\left(Z_{i}(\sigma, x, y)\right) d \sigma=s^{*}-s_{-}, \text {for } t>t^{*} .
\end{array}\right.
$$

We conduct numerical simulations for system (0.12), using the parameters in Table 1-3, and setting the diffusion coefficient $\varepsilon_{1}=0.01, \varepsilon_{2}=0.005$, in order to observe the growth and spread of adult population of the two species. The simulation is conducted in a $300 * 300$ square of the $x-y$ plane, as in the reference [19]. We set the random initial distribution for the two species by taking a random number at 25 random positions.

Next we plot the solutions of system (0.12) at several specified time in Figure 7. The $x$ - and $y$-axis describe the spatial coordinates, and the z-axis is the adult population number. In this 
figure we will observe the growth of the two species and the spread in space, and we can also see vividly that the model generates obvious species isolates after some time.
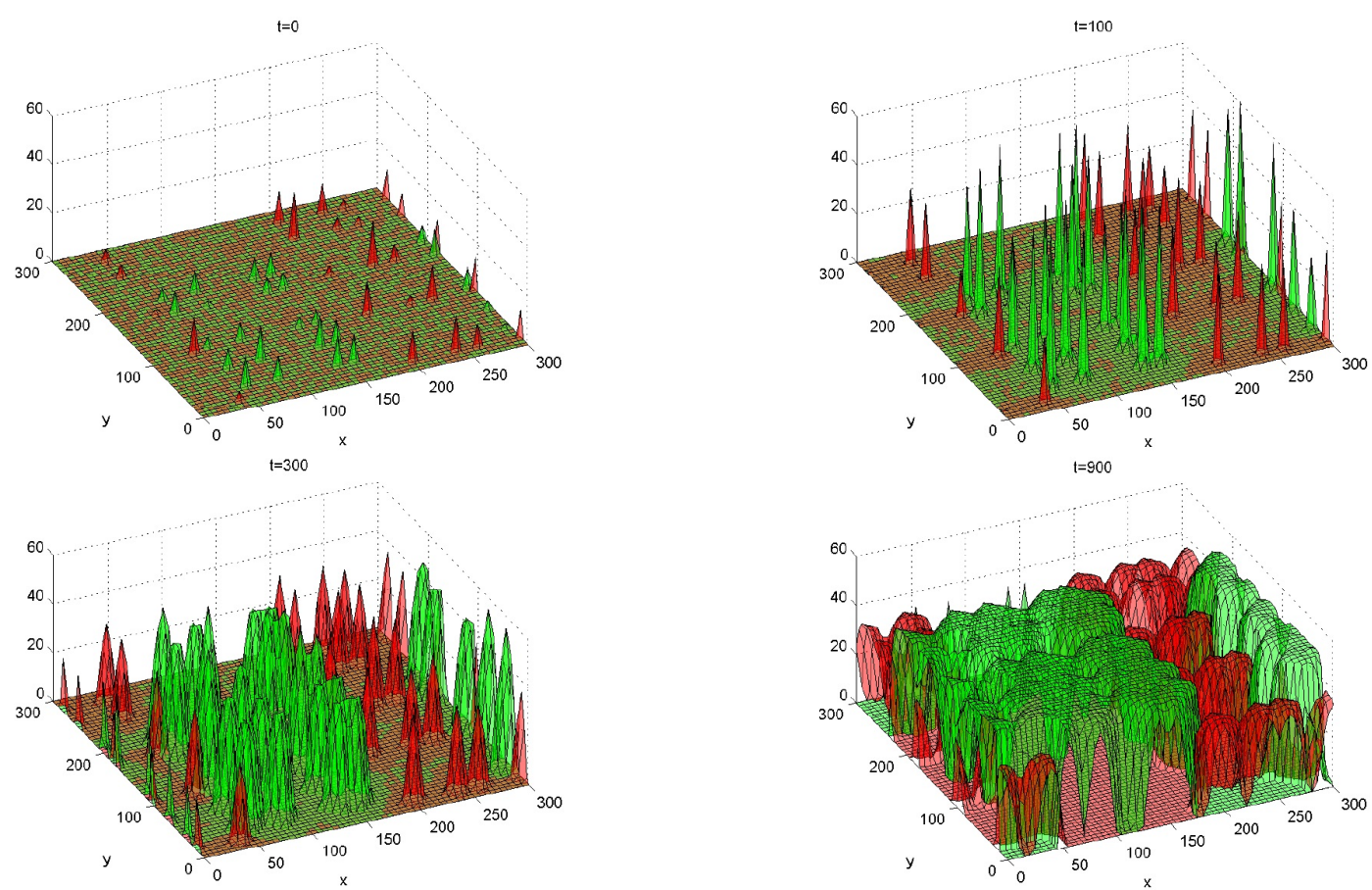

Figure 7: In this figure we show the numerical simulations for the spatial model (0.12), describing the spread of adult population of the two species. The red part represents species 1 American beech, and the green part represents species 2 eastern hemlock.

We also conduct the simulation for longer time, and we get the following results in Figure 8 . 

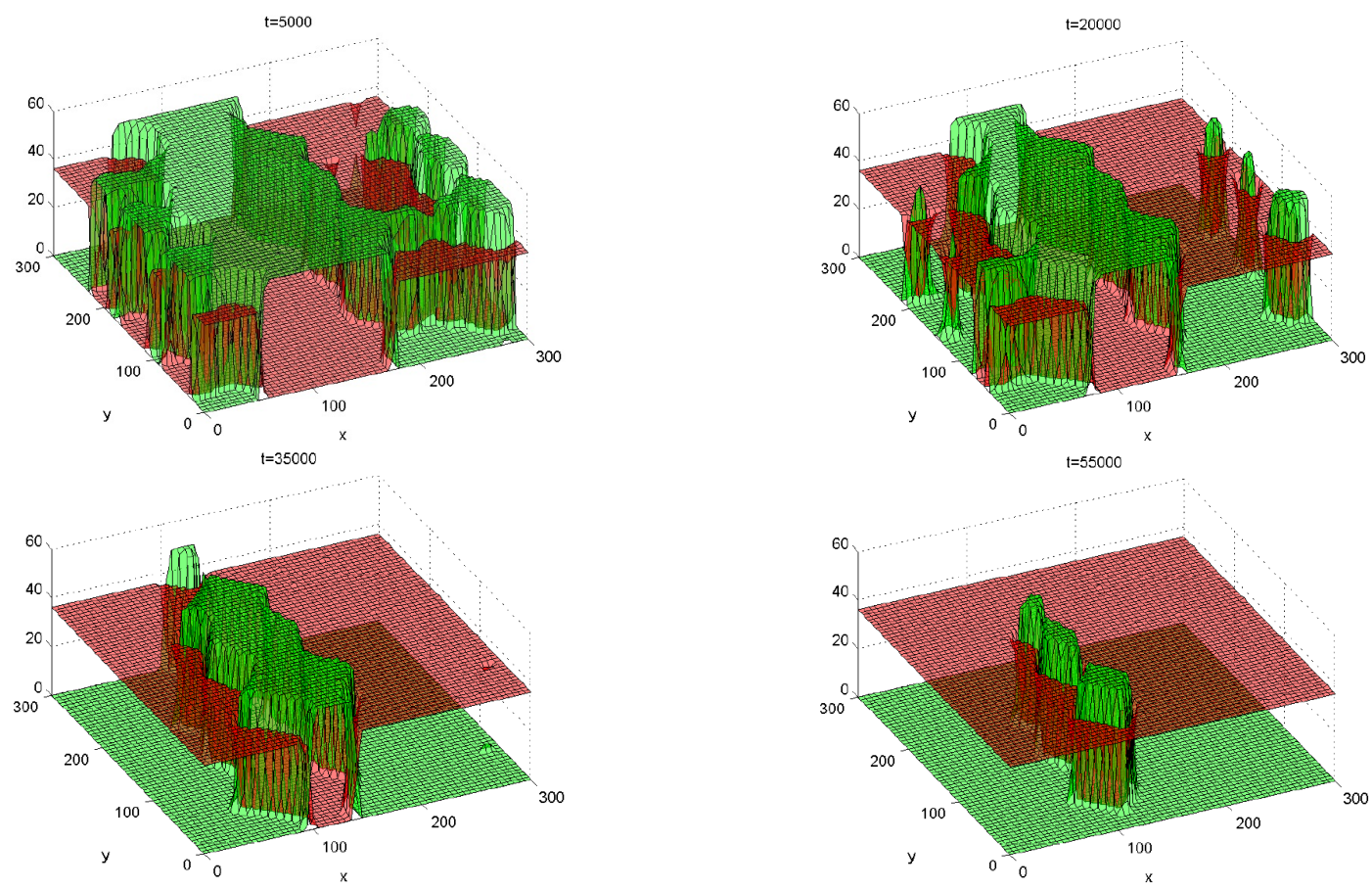

Figure 8: This figure shows the change of the distribution of adult population of two species in the long run. Notice that eastern hemlock(green) is disappearing and American beech(red) becomes dominant.

Summarizing all the figures above, we may conclude that eastern hemlock(green) grows and spreads faster than American beech(red) at first, but after long enough time, American beech begins to show its competency and gradually becomes the dominant species. This result also coincides with our previous result without considering the space in Figure 5.

Moreover, we plot the total population in the sample square for each species with respect to faster than American beech at first, and then it decreases. 


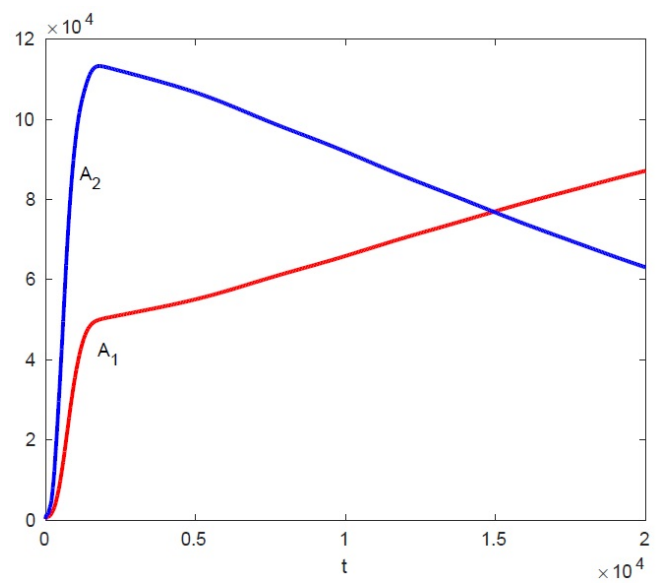

Figure 9: In this figure we show the total population in the $300 m \times 300 m$ square for each species in 20000 years. $A_{1}$ represents species 1 American beech, and $A_{2}$ represents species 2 eastern hemlock.

As in Figure 6, we can also observe the coexistence of both species in the spatial model. In Figure 10 we plot the long term distributions after the same change of parameters as in Figure 6.
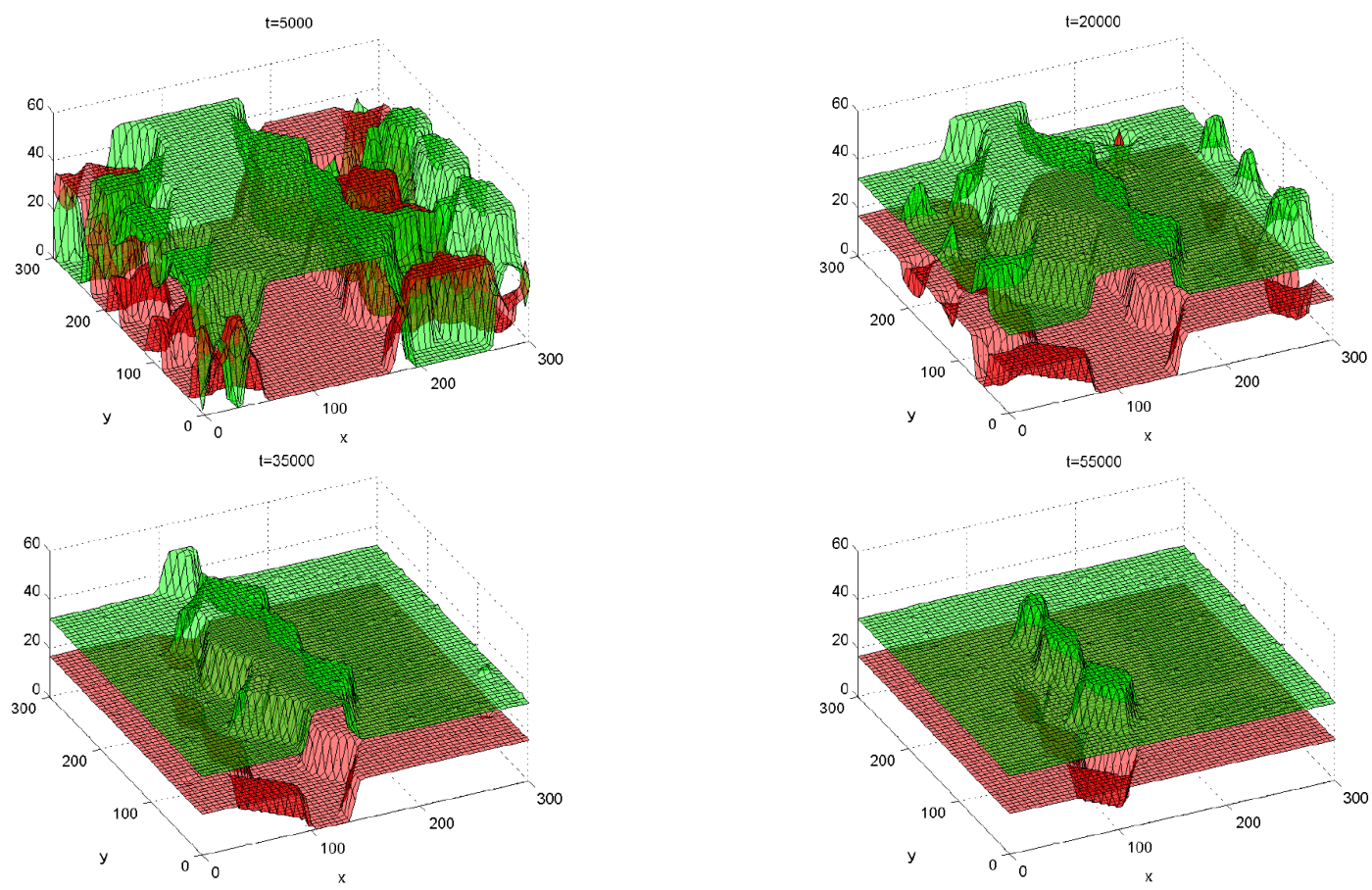

Figure 10: The longterm simulation for the spatial model with a change of the parameter $\zeta_{21}$ from 1.6 to 1. All the other parameters are the same as in Figure 8. We observe the coexistence of both species. 


\section{Discussion}

Studies of forest dynamics have a long history. There have been a large amount of researches by analyzing the existence of the interior coexistent equilibrium, we are able to establish a range 

have

$$
\begin{aligned}
\frac{d A(t)}{d t} & =\int_{s^{*}}^{+\infty} \partial_{t} u(t, s) d s=-f(A(t)) \int_{s^{*}}^{+\infty} \partial_{s} u(t, s) d s-\int_{s^{*}}^{+\infty} \mu(s) u(t, s) d s \\
& =f(A(t)) u\left(t, s^{*}\right)-\int_{s^{*}}^{+\infty} \mu(s) u(t, s) d s .
\end{aligned}
$$

Next we deal with the term $u\left(t, s^{*}\right)$. The characteristic curves for the first equation in (0.1) are (shown in Figure 11)

$$
\frac{d s(t)}{d t}=f(A(t))
$$




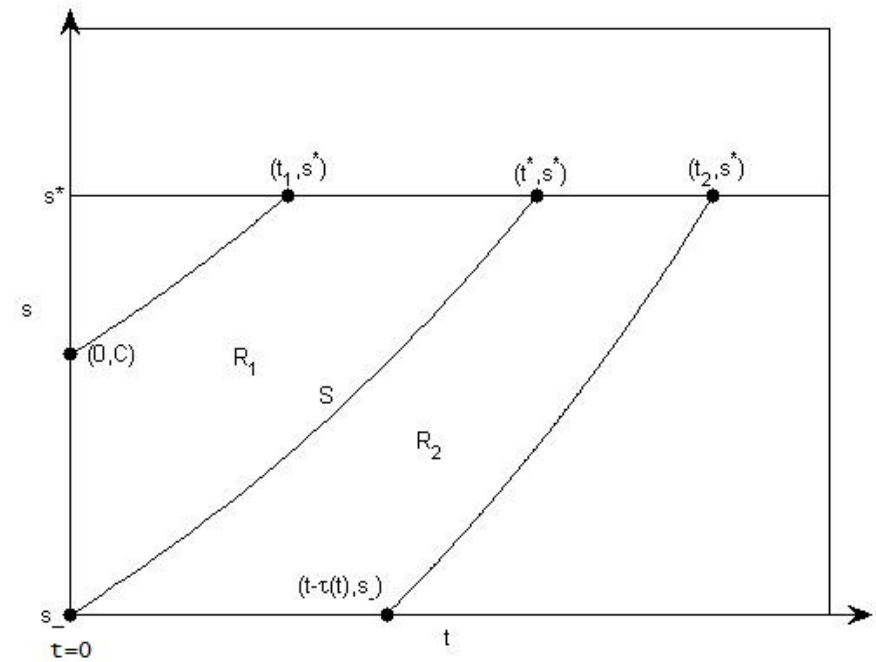

Figure 11: In this figure we present the characteristic curves (A.2).

Then we will have the following representation of $s$

$$
C+\int_{0}^{t} f(A(\sigma)) d \sigma=s(t)
$$

Suppose $t^{*}$ is the time when juveniles present at time 0 become adults, namely

$$
\int_{0}^{t^{*}} f(A(\sigma)) d \sigma=s^{*}-s_{-}
$$

We can see that the curve

$$
S=\left\{(t, s): 0 \leqslant t \leqslant t^{*}, s=s_{-}+\int_{0}^{t} f(A(\sigma)) d \sigma\right\}
$$

divides the strip $[0,+\infty) \times\left[s_{-}, s^{*}\right]$ into two parts $R_{1}$ and $R_{2}$. Assuming that $s-s_{-} \leqslant \int_{0}^{t} f(A(\sigma)) d \sigma$, then we can find $T(t, s) \geqslant 0$ such that

$$
\int_{t-T(t, s)}^{t} f(A(\sigma)) d \sigma=s-s_{-}
$$

in the region $R_{2}$, so it denotes the time it takes for a juvenile to grow to size $s$ at time $t$ from the minimal size $s_{-}$. Replacing $s$ in $u(t, s)$ with (A.3), we can compute formally as follows, assuming 
that $u$ is a $C^{1}$-function:

$$
\begin{aligned}
& \frac{d}{d t} u\left(t, C+\int_{0}^{t} f(A(\sigma)) d \sigma\right) \\
= & \partial_{t} u\left(t, C+\int_{0}^{t} f(A(\sigma)) d \sigma\right)+f(A(t)) \partial_{s} u\left(t, C+\int_{0}^{t} f(A(\sigma)) d \sigma\right) \\
= & -\mu\left(C+\int_{0}^{t} f(A(\sigma)) d \sigma\right) u\left(t, C+\int_{0}^{t} f(A(\sigma)) d \sigma\right) .
\end{aligned}
$$

This is a separable ODE with respect to $t$. Integration of this equation, and by using the initial distribution and the boundary condition, we obtain the following expression of $u(t, s)$

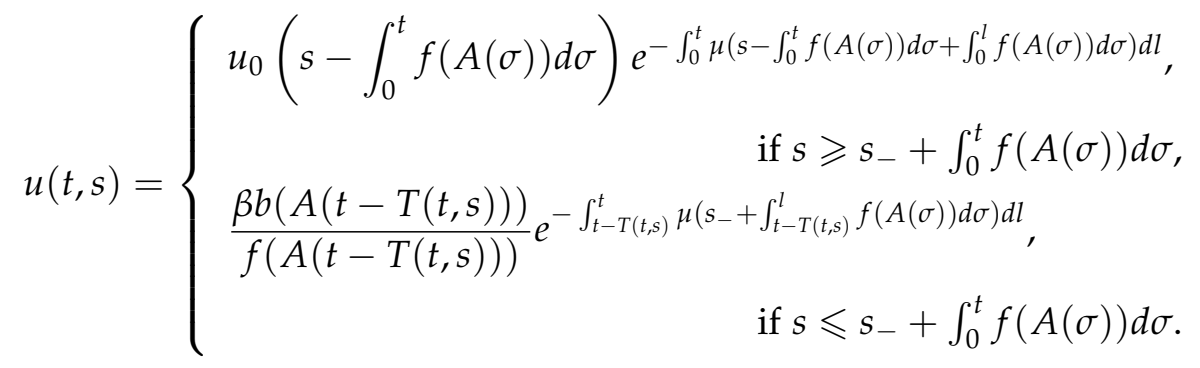

Whenever $s^{*}-s_{-} \leqslant \int_{0}^{t} f(A(\sigma)) d \sigma$, we can specifically define $\tau(t):=T\left(t, s^{*}\right)$ as the solution of

$$
\int_{t-\tau(t)}^{t} f(A(\sigma)) d \sigma=s^{*}-s_{-} .
$$

Actually the term $\tau(t)=T\left(t, s^{*}\right)$ represents the time spent by a newborn becoming an adult.

We now assume the mortality function as follows:

$$
\mu(s)=\left\{\begin{array}{l}
\mu_{A}>0, \text { if } s \geqslant s^{*}, \\
\mu_{J}>0, \text { if } s \in\left[s_{-}, s^{*}\right) .
\end{array}\right.
$$

Then when $s=s^{*}$, we have for $t \in\left[0, t^{*}\right]$,

$$
u\left(t, s^{*}\right)=u_{0}\left(s^{*}-\int_{0}^{t} f(A(\sigma)) d \sigma\right) e^{-\mu_{J} t},
$$

and for $t>t^{*}$,

$$
u\left(t, s^{*}\right)=\frac{\beta b(A(t-\tau(t)))}{f(A(t-\tau(t)))} e^{-\mu_{J} \tau(t)} .
$$

Replacing $u\left(t, s^{*}\right)$ back in (A.1), we get the model (0.5).

By differentiating the second equation of (0.5) in time, we obtain

$$
\frac{d}{d t} \int_{t-\tau(t)}^{t} f(A(\sigma)) d \sigma=0 \Leftrightarrow f(A(t))-f(A(t-\tau(t)))\left(1-\tau^{\prime}(t)\right)=0 .
$$


Therefore the state-dependent delay differential equation (0.6) is derived.

Remark A.1 Note that the function $t \rightarrow t-\tau(t)$ is strictly increasing because

$$
\frac{d}{d t}(t-\tau(t))=\frac{f(A(t))}{f(A(t-\tau(t)))}>0 .
$$

We conduct a comparison between the growth function (0.2) and the intrinsic function of the growth submodel in the simulator SORTIE. From the model (0.3), we only care about the growth of juveniles, so first we assume the radius function of a juvenile

$$
r(t)=\frac{\operatorname{diam}_{10}(t)}{2}
$$

where diam 10 represents the diameter at $10 \mathrm{~cm}$ height. We use the following change of variable to define the size $s$ which we are using in the model $(0.1)$

$$
s(t):=\ln \frac{r(t)}{r_{-}}
$$

where $r_{-}$is the minimal radius of the juvenile. We will have

$$
s^{\prime}(t)=\frac{r^{\prime}(t)}{r(t)}=f(A(t))
$$

Then the approximation of the derivative of $r(t)$, which describes the growth of the radius, is

$$
\frac{r(t+\Delta t)-r(t)}{\Delta t}=r(t) \cdot \frac{\alpha}{1+\delta A(t)}=r(t) \cdot \frac{\alpha A(t)^{-1}}{\delta+A(t)^{-1}} .
$$

Take $\Delta t=1$ (one year), then (A.9) shows the increase of the radius in one year.

On the other hand, we have the following formula for growth in SORTIE from $[19,8,18]$ :

$$
\text { Annual Radius Increase }=\text { Radius } \cdot \frac{G_{1} \cdot G L I}{\frac{G_{1}}{G_{2}}+G L I},
$$

where $G_{1}$ is the asymptotic growth rate at high light and $G_{2}$ is the slope at 0 or low light. The term GLI (global light index) describes the percentage of light transmitted through tree gaps and perceived by trees, thus is a measure for light. Comparing the two formulas (A.9) and (A.10), we find that they have the same form, and the parameters $A(t)^{-1}, \alpha, \delta$ correspond to GLI, $G_{1}, \frac{G_{1}}{G_{2}}$ 
respectively. So the choice of the growth function (0.2) is reasonable. Plus, this also explains what is size $s$ in our model (0.1). By this definition of $s(t)$, we have the minimal size of juveniles

$189 s_{-}=0\left(\right.$ as $\left.r(t)=r_{-}\right)$, and the minimal size of adults $s_{*}=\ln \frac{r^{*}}{r_{-}}$, where $r^{*}$ is the minimal radius of adults.

\section{Appendix B Positive equilibrium for two-species model}

We compute the positive equilibrium for the system (0.9), which is, we compute the solution for the following equations:

$$
\left\{\begin{array}{l}
0=e^{-\mu_{J_{1}} \tau_{1}} \beta_{1} A_{1}-\mu_{A_{1}} A_{1}, \\
\int_{t-\tau_{1}}^{t} \frac{\alpha_{1}}{1+\delta_{1}\left(\zeta_{11} A_{1}+\zeta_{12} A_{2}\right)} d \sigma=s^{*}-s_{-},
\end{array}\right.
$$

and

$$
\left\{\begin{array}{l}
0=e^{-\mu_{J_{2}} \tau_{2}} \beta_{2} A_{2}-\mu_{A_{2}} A_{2,} \\
\int_{t-\tau_{2}}^{t} \frac{\alpha_{2}}{1+\delta_{2}\left(\zeta_{21} A_{1}+\zeta_{22} A_{2}\right)} d \sigma=s^{*}-s_{-},
\end{array}\right.
$$

Obviously, $A_{1}=0, A_{2}=0$ is a trivial equilibrium for the species, in which case we have

$$
\tau_{1}=\frac{s^{*}-s_{-}}{\alpha_{1}}, \tau_{2}=\frac{s^{*}-s_{-}}{\alpha_{2}} .
$$

Moreover, we have two "boundary" equilibrium $\left(\bar{A}_{1}, 0\right)$ and $\left(0, \tilde{A}_{2}\right)$, where

$$
\begin{gathered}
\bar{A}_{1}=\frac{1}{\delta_{1} \zeta_{11}}\left(\frac{\alpha_{1}}{\mu_{J_{1}}\left(s^{*}-s_{-}\right)} \ln \frac{\beta_{1}}{\mu_{A_{1}}}-1\right), \\
\bar{\tau}_{1}=\frac{1}{\mu_{J_{1}}} \ln \frac{\beta_{1}}{\mu_{A_{1}}}, \bar{\tau}_{2}=\frac{\left(s^{*}-s_{-}\right)\left(1+\delta_{2} \zeta_{21} \bar{A}_{1}\right)}{\alpha_{2}},
\end{gathered}
$$

and

$$
\begin{gathered}
\tilde{A}_{2}=\frac{1}{\delta_{2} \zeta_{22}}\left(\frac{\alpha_{2}}{\mu_{J_{2}}\left(s^{*}-s_{-}\right)} \ln \frac{\beta_{2}}{\mu_{A_{2}}}-1\right), \\
\tilde{\tau}_{1}=\frac{\left(s^{*}-s_{-}\right)\left(1+\delta_{1} \zeta_{12} \tilde{A}_{2}\right)}{\alpha_{1}}, \tilde{\tau}_{2}=\frac{1}{\mu_{J_{2}}} \ln \frac{\beta_{2}}{\mu_{A_{2}}} .
\end{gathered}
$$

Now we turn to the positive equilibrium. As $A_{1}, A_{2} \neq 0$, we solve the first equation in (B.1) and (B.2) and get

$$
\tau_{1}=\frac{1}{\mu_{J_{1}}} \ln \frac{\beta_{1}}{\mu_{A_{1}}}, \quad \tau_{2}=\frac{1}{\mu_{J_{2}}} \ln \frac{\beta_{2}}{\mu_{A_{2}}}
$$


By the second equation of (B.1) and (B.2), we have

$$
\left\{\begin{array}{l}
\zeta_{11} A_{1}+\zeta_{12} A_{2}=\frac{1}{\delta_{1}}\left(\frac{\alpha_{1} \tau_{1}}{s^{*}-s_{-}}-1\right), \\
\zeta_{21} A_{1}+\zeta_{22} A_{2}=\frac{1}{\delta_{2}}\left(\frac{\alpha_{2} \tau_{2}}{s^{*}-s_{-}}-1\right)
\end{array}\right.
$$

We replace $\tau_{1}$ and $\tau_{2}$ in (B.4) by (B.3), and we get the following linear equations:

$$
\left\{\begin{array}{l}
\zeta_{11} A_{1}+\zeta_{12} A_{2}=\Phi_{1} \\
\zeta_{21} A_{1}+\zeta_{22} A_{2}=\Phi_{2}
\end{array}\right.
$$

where

$$
\Phi_{1}:=\frac{1}{\delta_{1}}\left[\frac{\alpha_{1}}{\mu_{J_{1}}\left(s^{*}-s_{-}\right)} \ln \frac{\beta_{1}}{\mu_{A_{1}}}-1\right], \quad \Phi_{2}:=\frac{1}{\delta_{2}}\left[\frac{\alpha_{2}}{\mu_{J_{2}}\left(s^{*}-s_{-}\right)} \ln \frac{\beta_{2}}{\mu_{A_{2}}}-1\right] .
$$

First, as we want a positive solution, we need the following conditions:

$$
\Phi_{1} \geqslant 0, \Phi_{2} \geqslant 0
$$

We solve the equation (B.5) directly without considering its solvability:

$$
A_{1}=\frac{\zeta_{22} \Phi_{1}-\zeta_{12} \Phi_{2}}{\zeta_{11} \zeta_{22}-\zeta_{12} \zeta_{21}}, \quad A_{2}=\frac{\zeta_{11} \Phi_{2}-\zeta_{21} \Phi_{1}}{\zeta_{11} \zeta_{22}-\zeta_{12} \zeta_{21}}
$$

In order to have a positive solution, we need the following conditions:

$$
\left\{\begin{array} { l } 
{ \zeta _ { 1 1 } \zeta _ { 2 2 } - \zeta _ { 1 2 } \zeta _ { 2 1 } > 0 , } \\
{ \zeta _ { 2 2 } \Phi _ { 1 } - \zeta _ { 1 2 } \Phi _ { 2 } > 0 , } \\
{ \zeta _ { 1 1 } \Phi _ { 2 } - \zeta _ { 2 1 } \Phi _ { 1 } > 0 , }
\end{array} \text { or } \left\{\begin{array}{l}
\zeta_{11} \zeta_{22}-\zeta_{12} \zeta_{21}<0 \\
\zeta_{22} \Phi_{1}-\zeta_{12} \Phi_{2}<0 \\
\zeta_{11} \Phi_{2}-\zeta_{21} \Phi_{1}<0
\end{array}\right.\right.
$$

or in another simplified form

$$
\frac{\zeta_{12}}{\zeta_{22}}<\frac{\Phi_{1}}{\Phi_{2}}<\frac{\zeta_{11}}{\zeta_{21}}, \quad \text { or } \quad \frac{\zeta_{11}}{\zeta_{21}}<\frac{\Phi_{1}}{\Phi_{2}}<\frac{\zeta_{12}}{\zeta_{22}}
$$

So we have

Lemma B.1 Under the condition (B.6) and (B.9), the equations (B.1) and (B.2) have a positive equilibrium as in (B.7). 
We check the conditions (B.6) and (B.9) for our previous results in Table 1-3, and we have

$$
\begin{aligned}
& \Phi_{1}=100.6839>0, \quad \Psi_{2}=133.4324>0, \\
& \frac{\zeta_{12}}{\zeta_{22}}=0.6, \quad \frac{\Phi_{1}}{\Phi_{2}}=0.7546, \quad \frac{\zeta_{11}}{\zeta_{21}}=0.625,
\end{aligned}
$$

which does not satisfy the condition (B.9), so there is no positive equilibrium in our previous simulation, and eastern hemlock is disappearing. In order to have a positive equilibrium, we reduce the influence of American beech towards eastern hemlock, namely we lower $\zeta_{21}$ from 1.6 to 1 . Then we have

$$
\frac{\zeta_{11}}{\zeta_{21}}=1
$$

which satisfies the condition (B.9). And we have the coexistence of both species as is shown in Figure 6 and Figure 10.

\section{References}

[1] Ó. Angulo, R. B. de la Parra, J. C. López-Marcos, and M. A. Zavala. Stand dynamics and tree coexistence in an analytical structured model: The role of recruitment. Journal of Theoretical Biology, 333:91-101, 2013.

[2] D. B. Botkin. Forest dynamics: An ecological model. Oxford University Press, 1993.

[3] D. B. Botkin, J. F. Janak, and J. R. Wallis. Some ecological consequences of a computer model of forest growth. Journal of Ecology, 60:849-872, 1972.

[4] M. Cammarano. Co-dominance and succession in forest dynamics: The role of interspecific differences in crown transmissivity. Journal of Theoretical Biology, 285:46-57, 2011.

[5] A. Cheaiib, A. Mollier, S. Thunot, C. Lambrot, S. Pellerin, and D. Loustau. Interactive effects of phosphorus and light availability on early growth of maritime pine seedlings. Annals of Forest Science, 62:575-583, 2005. 
[6] A. M. de Roos and L. Persson. Competition in size-structured populations: mechanisms inducing cohort formation and population cycles. Theoretical Population Biology, 63:1-16, 2003.

[7] A. Ducrot. Travelling waves for a size and space structured model in population dynamics: Point to sustained oscillating solution connections. Journal of Differential Equations, 250:410-449, 2011.

[8] R. K. Kobe, S. W. Pacala, J. A. Silander Jr., and C. D. Canham. Juvenile tree survivorship as a component of shade tolerance. Ecological Applications, 5(2):517-532, 1995.

[9] P. Köhler and A. Huth. An individual based rain forest model - concepts and simulation results. In A. Kastner-Maresch, W. Kurth, M. Sonntag, and B. Breckling, editors, Individual-based structural and functional models in ecology, volume 52 of Bayreuther Forum Ökologie, pages 35-51. Bayreuther Institut für terrestrische Ökosystemforschung, Bayreuth, 1998.

[10] T. S. Kohyama and T. Takada. One-sided competition for light promotes coexistence of forest trees that share the same adult height. Journal of Ecology, 100:1501-1511, 2012.

[11] T. E. Kolb, K. C. Steiner, L. H. McCormick, and T. W. Bowersox. Growth response of northern red-oak and yellow-poplar seedlings to light, soil moisture and nutrients in relation to ecological strategy. Forest Ecology and Management, 38:65-78, 1990.

[12] K. Kramer, I. Leinonen, and D. Loustau. The importance of phenology for the evaluation of impact of climate change on growth of boreal, temperate and mediterranean forests ecosystems: an overview. International Journal of Biometeorology, 44:67-75, 2000.

[13] S. A. Levin, B. Grenfell, A. Hastings, and A. S. Perelson. Mathematical and computational challenges in population biology and ecosystems science. Science, 275:334-343, 1997. 
[14] J. Liu and P. S. Ashton. Individual-based simulation models for forest succession and management. Forest Ecology and Management, 73:157-175, 1995.

[21] A. Porté and H. H. Bartelink. Modelling mixed forest growth: a review of models for forest management. Ecological Modelling, 150:141-188, 2002. three geographically separate origins of maritime pine (pinus pinaster) under saline conditions. Tree Physiology, 15:569-576, 1995.

[16] P. Magal and S. Ruan. Center manifolds for semilinear equations with non-dense domain and applications to hopf bifurcation in age structured models. In AMS eBook Collections, volume 202 of Memoirs of the American Mathematical Society. American Mathematical Society, Providence, Rhode Island, 2009.

[17] N. L. E. Obiang, A. Ngomanda, O. Hymas, É. Chézeauxl, and N. Picard. Diagnosing the demographic balance of two light-demanding tree species populations in central africa from their diameter distribution. Forest Ecology and Management, 313:55-62, 2014.

[18] S. W. Pacala, C. D. Canham, J. Saponara, J. A. Silander Jr., R. K. Kobe, and E. Ribbens. Forest models defined by field measurements: estimation, error analysis and dynamics. Ecological Monographs, 66(1):1-43, 1996.

[19] S. W. Pacala, C. D. Canham, and J. A. Silander Jr. Forest models defined by field measurements: I. the design of a northeastern forest simulator. Canadian Journal of Forest Research, 23:1980-1988, 1993.

[20] S. W. Pacala, C. D. Canham, J. A. Silander Jr., and R. K. Kobe. Sapling growth as a function of resources in a north temperate forest. Canadian Journal of Forest Research, 24:2172-2183, 1994.

[15] D. Loustau, S. Crepeau, M. G. Guye, M. Sartore, and E. Saur. Growth and water relations of 
[22] E. Ribbens, J. A. Silander Jr., and S. W. Pacala. Seedling recruitment in forests: calibrating models to predict patterns of tree seedling dispersion. Ecology, 75(6):1794-1806, 1994.

[23] W. E. Ricker. Stock and recruitment. Journal of the Fisheries Research Board of Canada, 11(5):559-623, 1954.

[24] W. E. Ricker. Computation and interpretation of biological studies of fish populations. In Bulletin of the Fisheries Research Board of Canada, volume 191. Fisheries and Marine Service, Ottawa, 1975.

[25] H. H. Shugart and D. C. West. Development of an appalachian deciduous forest succession model and its application to assessment of the impact of the chestnut blight. Journal of Environmental Management, 5:161-179, 1977.

[26] H. L. Smith. Reduction of structured population models to threshold-type delay equations and functional differential equations: a case study. Mathematical Biosciences, 113:1-23, 1993.

[27] H. L. Smith. A structured population model and a related functional differential equation: global attractors and uniform persistence. Journal of Dynamics and Differential Equations, 6(1):71-99, 1994.

[28] N. Strigul, D. Pristinski, D. Purves, J. Dushoff, and S. Pacala. Scaling from trees to forests: tractable macroscopic equations for forest dynamics. Ecological Monographs, 78(4):523-545, 2008.

[29] R. M. Teck and D. E. Hilt. Individual-tree diameter growth model for the northeastern united states. Research paper NE-649, US. Department of Agriculture, Forest Service, Northeastern Forest Experiment Station, Radnor, PA, 1991.

[30] P. Trichet, D. Loustau, C. Lambrot, and S. Linder. Manipulating nutrient and water availability in a maritime pine plantation: effects on growth, production, and biomass allocation at canopy closure. Annals of Forest Science, 65(8):814, 2008. 
[32] M. A. Zavala, Ó. Angulo, R. B. de la Parra, and J. C. López-Marcos. An analytical model of stand dynamics as a function of tree growth, mortality and recruitment: The shade tolerance-stand structure hypothesis revisited. Journal of Theoretical Biology, 244:440-450, 2007. 


\section{Tables}

\begin{tabular}{cccc}
\hline Parameter & Interpretation & value & Reference \\
\hline$\mu_{J_{1}}$ & natural mortality rate for juveniles & 0.03 & estimated \\
$\mu_{A_{1}}$ & natural mortality rate for adults & 0.001 & estimated \\
$\beta_{1}$ & birth rate in absence of birth limitation & 2 & estimated \\
$s_{-}$ & minimal size for juvenile & 0 & {$[19]$} \\
$s^{*}$ & minimal size for adult & $\ln 50$ & [22] \\
$\xi_{1}$ & parameter in the Ricker type function & 0 & estimated \\
$\tau_{01}$ & time delay of the juveniles present & 121 & estimated \\
& at time 0 to become adults & & \\
$\alpha_{1}$ & growth rate of juveniles without adults & 0.1709 & computed \\
$\delta_{1}$ & parameter describing the descending speed & & \\
& of the growth ratewhen adult population increases & 0.1 & estimated \\
\hline
\end{tabular}

Table 1: Parameter values of the best fit for species 1: American beech. 


\begin{tabular}{cccc}
\hline Parameter & Interpretation & value & Reference \\
\hline$\mu_{J_{2}}$ & natural mortality rate for juveniles & 0.031 & estimated \\
$\mu_{A_{2}}$ & natural mortality rate for adults & 0.0037 & estimated \\
$\beta_{2}$ & birth rate in absence of birth limitation & 4 & estimated \\
$s_{-}$ & minimal size for juvenile & 0 & {$[19]$} \\
$s^{*}$ & minimal size for adult & $\ln 50$ & {$[22]$} \\
$\xi_{2}$ & parameter in the Ricker type function & 0 & estimated \\
$\tau_{02}$ & time delay of the juveniles present & 127 & estimated \\
& at time 0 to become adults & & \\
$\alpha_{2}$ & growth rate of juveniles without adults & 0.249 & computed \\
$\delta_{2}$ & parameter describing the descending speed & & \\
& of the growth rate when adult population increases & 0.1 & estimated \\
\hline
\end{tabular}

Table 2: Parameter values of the best fit for species 2: eastern hemlock. 


\begin{tabular}{cccc}
\hline Parameter & Interpretation & value & Reference \\
\hline$\zeta_{11}$ & parameter in the competition term describing & & \\
& the intraspecific competition among American beech & 1 & estimated \\
$\zeta_{12}$ & parameter in the competition term describing the & & \\
& interspecific influence of eastern hemlock on American beech & 0.6 & estimated \\
$\zeta_{21}$ & parameter in the competition term describing the & & \\
& interspecific influence of American beech on eastern hemlock & 1.6 & estimated \\
$\zeta_{22}$ & parameter in the competition term describing & & \\
& the intraspecific competition among eastern hemlock & 1 & estimated \\
$\tau_{01}$ & time delay of the American beech juveniles & 201 & estimated \\
& time delay of the eastern hemlock juveniles & & \\
$\tau_{02}$ & present at time 0 to become adults & 208 & estimated
\end{tabular}

Table 3: Other parameter values for two-species model. 